\title{
REBOISEMENT DE FRICHES CALCAIRES DANS LE CENTRE DE LA FRANCE : CHOIX DES ESSENCES INFLUENCE DE L'AGE DES PLANTS
}

\author{
C.T.G.R.E.F. - Nogent/Vernisson \\ Compte-rendu d'expérience no 19735
}

Class. Oxford $232.11: 232.411 .2: 114.465$ (44 CENTRE)

Résumé : Une expérimentation installée de 1953 à 1955 sur le Domaine des Barres, au sud du Bassin Parisien, met en évidence l'intérêt de divers pins du groupe nigra pour le reboisement des friches calcaires; les plants de 3 ans (1-2) se sont avérés supérieurs aux plants plus jeunes.

\section{OBJET DE L'EXPÉRIMENTATION}

Les expériences instaliées de 1953 à 1955 sur le Domaine des Barres avaient pour but :

- de déterminer les essences qui, grâce à des techniques mécanisées d'installation, s'avèreraient les meilleures pour le boisement des friches calcaires du Centre de la France ;

- de comparer, pour les mêmes conditions de reboisement, le comportement de plants d’âges différents.

\section{CONDITIONS}

Le Domaine des Barres est situé à $130 \mathrm{~km}$ au sud de Paris, au voisinage de la ligne de partage des eaux entre les vallées de la Seine et de la Loire. Son climat est donc celui des plaines du centre de la France, avec de larges influences ligériennes : température moyenne voisine de $10^{\circ}$ avec des gelées tardives assez fréquentes; pluviosité annuelle $670 \mathrm{~mm}$ (pouvant descendre jusqu'à $450 \mathrm{~mm}$ ) relativement bien répartie avec, toutefois, des printemps assez secs. Le sol (1). sur la zone qui nous intéresse, est une rendzine grise de pelouse sur calcaire de Beauce, caractérisée par:

- une forte proportion de calcaire actif entraînant un pH élevé voisin de 8 ,

- un $\mathrm{C} / \mathrm{N}$ voisin de 10 (plus faible en surface).

- une capacité d'échange relativement faible pour une rendzine et un complexe absorbant saturé.

La végétation est essentiellement une pelouse xérocálciphile (Festuca duriuscula, Carex glauca, Brachypodium pinnatum, ...) avec une strate arbustive clairsemée (Juniperus communis, Cornus sanguinea, ...).

\section{DESCRIPTION DES EXPÉRIENCES}

Toutes les expériences ont été installées après un travail du sol par bandes décapées et soussolées distantes de 1,50 m (charrue décapeuse Jardin + sous soleuse agricole Fondeur).

Elles comportent toutes un dispositif statistique en blocs complets avec 4,3 ou 2 répétitions. sur des placeaux de 150 à 300 plants, à l'écartement $1,50 \times 1,50 \mathrm{~m}$ (4.400 plants par hectare).

(1) Voir: DUCHAUFOUR, Pédologie, tableaux descriptifs et analytiques des sols, page 40. Ecole Nationale des Eaux et Forêts, Nancy 1957. 


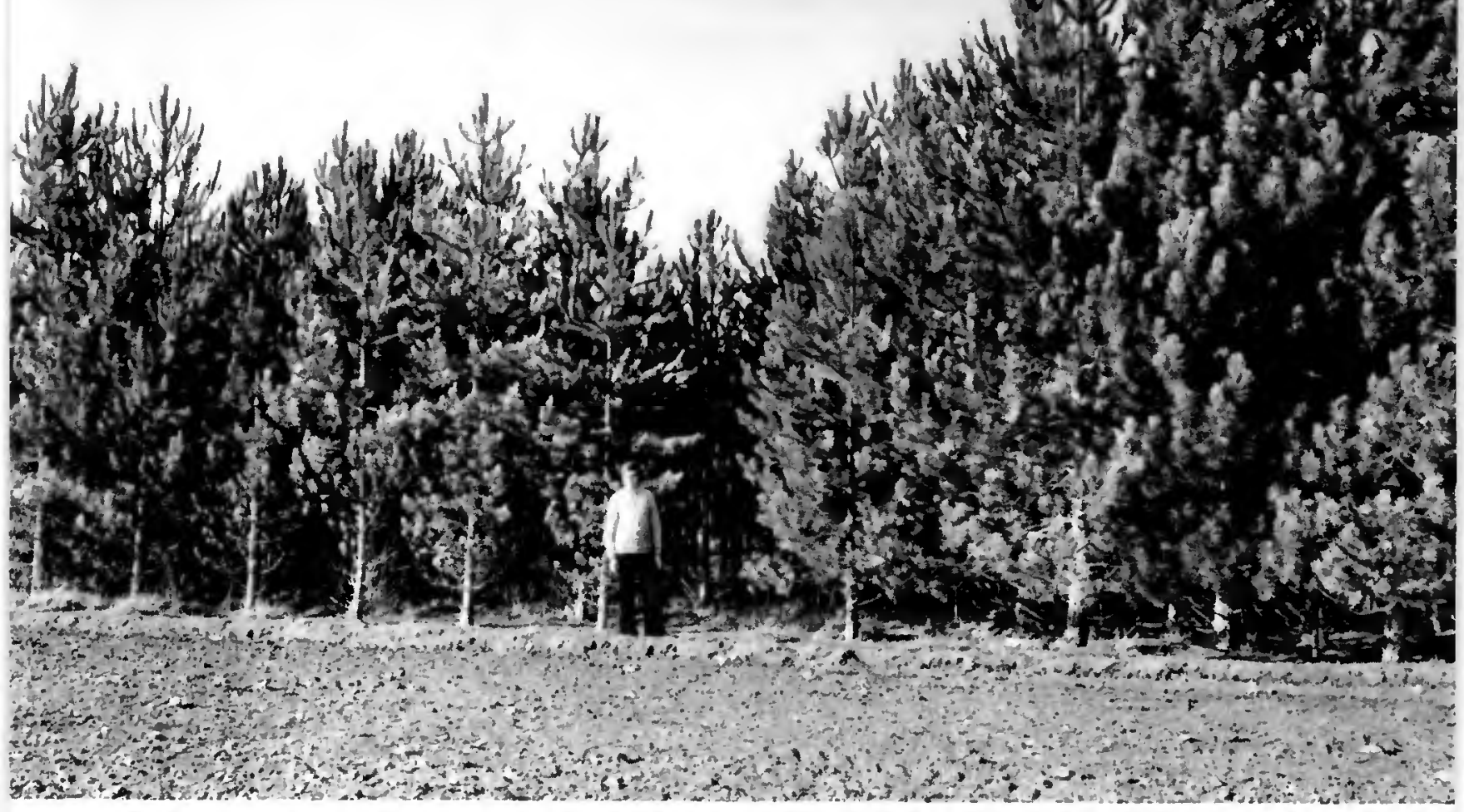

Expérience G2. A droite, Pins laricios de Calabre 1.2; à gauche, Pins lariclos de Corse 1.1

Photo DIMIER.VALLET

- La première expérience (G-2) installée à l'automne 1953, faisait appel à une grande variété d'essences:

Pin noir d'Autriche (Pinus nigra Arn. var. austriaca Endl.)

Pin laricio de Corse (Pinus nigra Arn. var. corsicana Loud.)

Pin laricio de Calabre (Pinus nigra Arn. var. calabrica Delam.)

Pin de Pallas (Pinas nigra Arn. var. pallasiana Lamb.)

Pin de Salzmann (Pinus nigra Arn. var. salzmanni Dunal.)

Pin sylvestre (Pinus sylvestris L.)

Cèdre de l'Atlas (Cedrus atlantica Man.)

Cyprès de l'Arizona (Cupressus glabra Sudworth) Epicéa omorica (Picea omorica Pancic)

Mélèze d'Europe (Larix decidua Mill.)

Douglas bleu (Pseudotsuga glauca Mayr.)
1-2 Provenance Ardennes

1-3 Provenance Les Barres

1-O Provenance Les Barres

1-1 Provenance Corse

1-0 Provenance Les Barres

1-1 Provenance Calabre

1-2 Provenance Calabre

1-3 Provenance Les Barres

1-O Provenance Les Barres

1-O Provenance diverses

1-O Provenance Les Barres race des

Causses

1-0 Provenance diverses

1-0 Provenance Valbonne

1-1 Provenance Les Barres

1-O Provenance Embrun +

Tyrol

autrichien

1-1 Provenance Les Barres

1-2 Provenance Les Barres 
- La deuxième expérience (E-3), installée à l'automne 1954, permettait de comparer des plants de pin d'âges différents :

Pin laricio de Corse (Pinus nigra Arn. var. corsicana Loud.)

Pin laricio de Calabre (Pinus nigra Arn. var. calabrica Delam.)

Pin de Pallas (Pinus nigra Arn. var. pallasiana Lamb.)

$\begin{array}{ll}\text { 1-0 Provenance } & \begin{array}{l}\text { Sécherie de } \\ \text { Marghèse (2) }\end{array} \\ \text { 1-1 Provenance Corte } \\ \text { 1-2 Provenance Corte } \\ \text { 1-0 Provenance Calabre } \\ \text { 1-1 Provenance Calabre } \\ \text { Les Barres } \\ \text { 1-2 Provenance Calabre } \\ \text { 1-0 Provenance Les Barres } \\ \text { 1-1 Provenance Les Barres }\end{array}$

- Enfin, la troisième expérience (BR-3), mise en place à l'automne 1955, permettait la comparaison des plants $1-2$ des essences suivantes:

Pin noir d'Autriche (Pinus nigra Arn. var, austriaca Endl.)

Pin laricio de Corse (Pinus nigra Arn. var. corsicana Loud.)

Pin laricio de Calabre (Pinus nigra Arn. var. calabrica Delam.)

Pin de Pallas (Pinus nigra Arn. var. pallasiana Lamb.)

Provenance Thessalie

Provenance Les Barres

Provenance Les Barres

Provenance Les Barres

(2) La provenance exacte n'est pas connue.

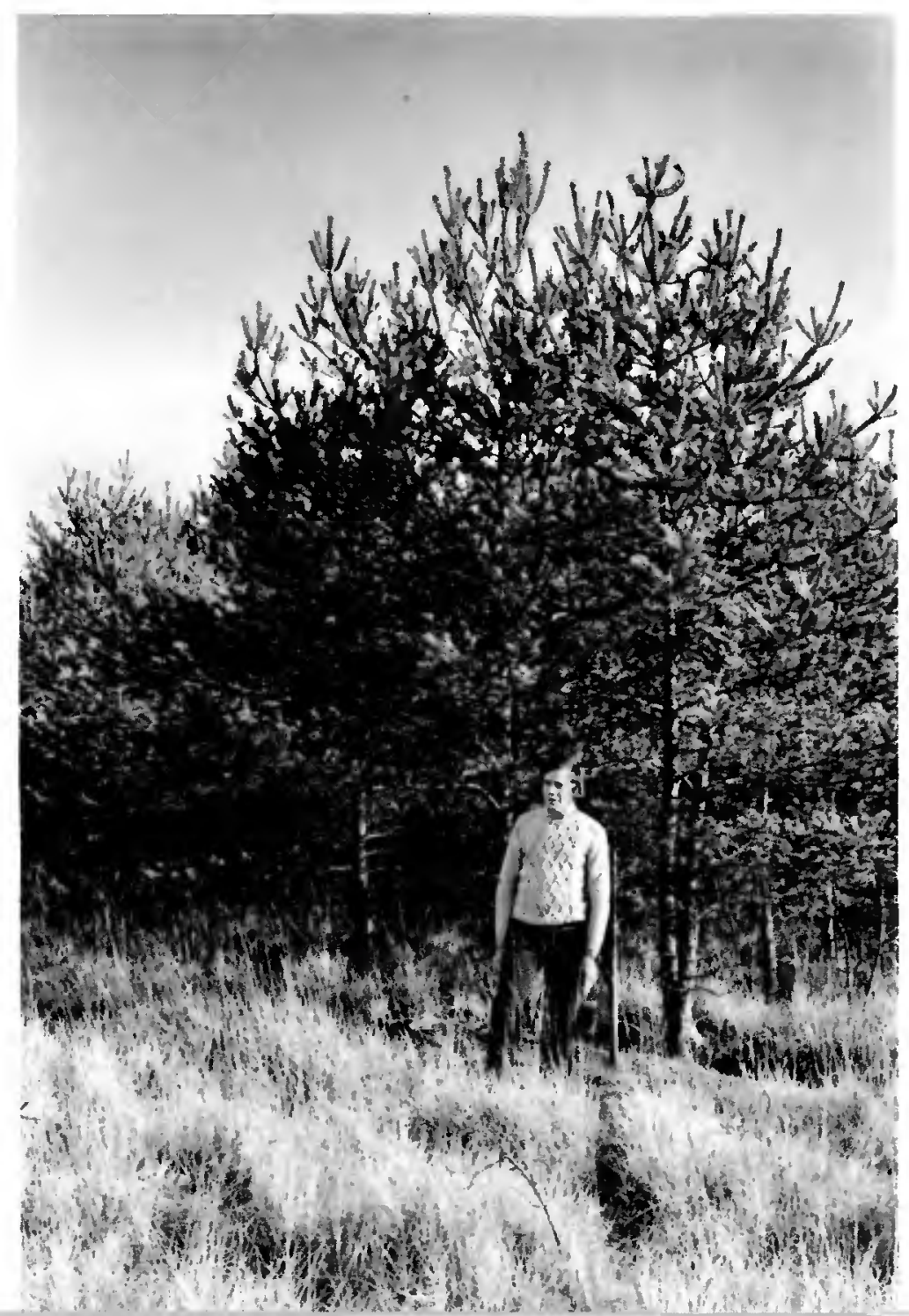

Pins sylvestres, race des Causses

hoto DIMIER-VALLET 
Les deux dernières expériences ont fait l'objet de regarnis relativement importants pour certaines essences (mortalité due en grande partie aux gelées exceptionnelles de février 1956).

\section{RÉSULTATS}

Les observations suivantes ont été faites :

- Automne 1966, comptage de survie et mesure des hauteurs,

- Mai 1971, comptage de survie, mesure des circonférences à 1,30 m et des hauteurs.

Les résultats sont consignés dans le tableau ci-après:

\begin{tabular}{|c|c|c|c|c|c|c|c|c|c|}
\hline \multirow[b]{2}{*}{$\begin{array}{l}\text { Expé- } \\
\text { rience }\end{array}$} & \multirow{2}{*}{\multicolumn{2}{|c|}{$\begin{array}{l}\text { Plants mis } \\
\text { en place }\end{array}$}} & \multirow[b]{2}{*}{$\begin{array}{c}\% \\
\text { total } \\
\text { regarnis }\end{array}$} & \multicolumn{2}{|c|}{ Observat. 1966} & \multicolumn{4}{|c|}{ Observations 1971} \\
\hline & & & & $\begin{array}{c}\% \\
\text { survie } \\
*\end{array}$ & $\begin{array}{l}\text { Hauteur } \\
\text { moyenne } \\
\text { en m }\end{array}$ & $\begin{array}{c}\% \\
\text { survie } \\
*\end{array}$ & $\begin{array}{c}\text { Hauteur } \\
\text { movenne } \\
(\mathrm{mm})\end{array}$ & $\begin{array}{c}\text { Circon- } \\
\text { férence } \\
\text { moyenne } \\
(\mathrm{mm})\end{array}$ & $\begin{array}{c}\text { Surface } \\
\text { terrière } \\
\text { moyenne } \\
\left(\mathrm{m}^{2} / \mathrm{ha}\right)\end{array}$ \\
\hline \multirow{5}{*}{ 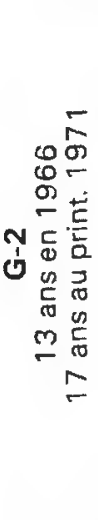 } & $\begin{array}{l}\text { Pin noir } \\
\text { d'Autriche }\end{array}$ & $\begin{array}{l}1-2 \\
1-3\end{array}$ & $\begin{array}{l}0 \\
0\end{array}$ & $\begin{array}{l}90 \\
88\end{array}$ & $\begin{array}{l}1,40 \\
1.80\end{array}$ & $\begin{array}{l}86 \\
85\end{array}$ & $\begin{array}{l}3,52 \\
3,21\end{array}$ & $\begin{array}{l}184 \\
149\end{array}$ & $\begin{array}{l}95 \\
54\end{array}$ \\
\hline & $\begin{array}{l}\text { Pin laricio } \\
\text { de Calabre }\end{array}$ & $\begin{array}{l}1-0 \\
1-1 \\
1-2 \\
1-3\end{array}$ & $\begin{array}{l}0 \\
0 \\
0 \\
0\end{array}$ & $\begin{array}{l}48 \\
80 \\
80 \\
74\end{array}$ & $\begin{array}{l}0,82 \\
1,40 \\
1,40 \\
2,08\end{array}$ & $\begin{array}{c}\text { Disparu } \\
66 \\
57 \\
72\end{array}$ & $\begin{array}{c}- \\
2,67 \\
3,57 \\
3,63\end{array}$ & $\begin{array}{l}1 \overline{61} \\
201 \\
195\end{array}$ & $\begin{array}{r}- \\
55 \\
86 \\
110\end{array}$ \\
\hline & $\begin{array}{l}\text { Pin laricio } \\
\text { de Corse }\end{array}$ & $\begin{array}{l}1-0 \\
1-1\end{array}$ & $\begin{array}{l}0 \\
0\end{array}$ & $\begin{array}{l}57 \\
93\end{array}$ & $\begin{array}{l}0,72 \\
1,42\end{array}$ & $\begin{array}{l}29 \\
93\end{array}$ & $\begin{array}{l}2,42 \\
3,30\end{array}$ & 159 & ** \\
\hline & $\begin{array}{l}\text { Pin de } \\
\text { Pallas }\end{array}$ & $1-0$ & 0 & 84 & 1,12 & 42 & 2,72 & $* *$ & $* *$ \\
\hline & $\begin{array}{l}\text { Pin } \\
\text { sylvestre }\end{array}$ & $1-0$ & 40 & $* *$ & $* *$ & 60 & 2,58 & $* *$ & $* *$ \\
\hline \multirow{3}{*}{ 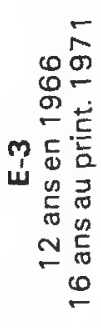 } & $\begin{array}{l}\text { Pin laricio } \\
\text { de Calabre }\end{array}$ & $\begin{array}{l}1-0 \\
1-1 \\
1-2\end{array}$ & $\begin{array}{l}31 \\
46 \\
15\end{array}$ & $\begin{array}{l}54 \\
55 \\
79\end{array}$ & $\begin{array}{l}1,56 \\
2,17 \\
1,98\end{array}$ & $\begin{array}{l}44 \\
50 \\
75\end{array}$ & $\begin{array}{l}3,15 \\
3,65 \\
3,81\end{array}$ & $\begin{array}{c}* * \\
194 \\
218\end{array}$ & $\begin{array}{c}* * \\
68 \\
128\end{array}$ \\
\hline & $\begin{array}{l}\text { Pin laricio } \\
\text { de Corse }\end{array}$ & $\begin{array}{l}1-0 \\
1-1 \\
1-2\end{array}$ & $\begin{array}{l}75 \\
21 \\
13\end{array}$ & $\begin{array}{l}18 \\
69 \\
63\end{array}$ & $\begin{array}{l}1,25 \\
1,83 \\
2,04\end{array}$ & $\begin{array}{c}21 * * * \\
66 \\
66 * * *\end{array}$ & $\begin{array}{l}2,54 \\
3,78 \\
3,90\end{array}$ & $\begin{array}{c}* * \\
162 \\
192\end{array}$ & $\begin{array}{l}* * \\
60 \\
81\end{array}$ \\
\hline & $\begin{array}{l}\text { Pin de } \\
\text { Pallas }\end{array}$ & $\begin{array}{l}1-0 \\
1-1\end{array}$ & $\begin{array}{l}47 \\
25\end{array}$ & $\begin{array}{l}36 \\
80\end{array}$ & $\begin{array}{l}1,68 \\
2,23\end{array}$ & $\begin{array}{l}36 \\
79\end{array}$ & $\begin{array}{l}3,47 \\
4,72\end{array}$ & $\stackrel{* *}{205}$ & $\stackrel{* *}{123}$ \\
\hline \multirow{4}{*}{ 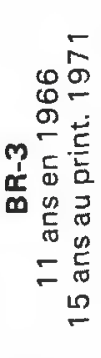 } & $\begin{array}{l}\text { Pin noir } \\
\text { d'Autriche }\end{array}$ & & 17 & 88 & 1,86 & 85 & 3,71 & 147 & 67 \\
\hline & $\begin{array}{l}\text { Pin laricio } \\
\text { de Calabre }\end{array}$ & $1-2$ & 3 & 100 & 3,18 & 90 & 5,52 & 234 & 171 \\
\hline & $\begin{array}{l}\text { Pin laricio } \\
\text { de Corse }\end{array}$ & $1-2$ & 61 & 70 & 2,64 & 67 & 5,00 & 211 & 112 \\
\hline & $\begin{array}{l}\text { Pin de } \\
\text { Pallas }\end{array}$ & $1-2$ & 5 & 100 & 2,94 & 91 & 5,08 & 205 & 140 \\
\hline
\end{tabular}

- Calculée par rapport au nombre de plants de la plantation initiale, sans tenir compte des regarnis éventuels. * Mesures non effectuées, ou valeur impossible à calculer faute de données.

* * Résultats s'expliquant vraisemblablement par des omissions lors des comptages de 1966. 


\section{INTERPRÉTATION}

L'interprétation est rendue difficile par le grand nombre de modalités comparées dans les deux premières expériences et par le fait qu'il n'a pas toujours été possible d'utiliser des plants de même provenance. Sous ces réserves, un certain nombre de résultats intéressants peuvent être dégagés :

La première expérience G-2, peut être considérée comme un test d'élimination pour le choix des essences; ses résultats ne sont donnés que pour les pins du groupe nigra (Salzmann excepté) et les pins sylvestres; les autres essences ont totalement disparu (mélèze, douglas) ou présentent un nombre très réduit de survivants d'aspect rabougri (pin de Salzmann, cèdre, Picea omorica). Cet échec ne doit cependant pas être interprété d'une manière trop absolue pour les essences utilisées seulement en 1-0 (pin de Salzmann, cèdre, mélèze), les autres expériences mettant en évidence le mauvais comportement des plants de cet âge.

Le pin sylvestre, utilisé lui aussi en 1-0 seulement, a une croissance faible et surtout une forme extrêmement mauvaise, ce qui peut s'expliquer par la provenance.

En définitive, les seules essences pouvant être retenues sont les pins du groupe nigra.

Les expériences E-3 et BR-3 permettent de comparer les variétés du groupe nigra.

Le pin noir d'Autriche a une survie satisfaisante mais une croissance inférieure à celle des autres variétés.

Le laricio de Corse a une reprise difficile et s'est montré sensible aux froids de 1956, mais il présente une bonne croissance en hauteur et en circonférence; la surface terrière est influencée par le taux de survie un peu plus faible.

Le laricio de Calabre et le pin de Pallas se classent en tête.

Enfin, l'expérience E-3 permet d'apprécier l'influence de l'âge des plants : l'échec est quasi général avec les 1-0. Les 1-1 permettent d'assurer la réussite du boisement mais s'avèrent systématiquement inférieurs aux 1-2 pour la croissance.

\section{APPLICATIONS PRATIQUES}

\section{Travail du sol}

Les expériences décrites dans le compte rendu ne permettent pas de tester l'effet du travail du sol, mais la comparaison avec les plantations voisines confirme ses bienfaits. La technique pourrait cependant être améliorée : l'intérêt du sous-solage est indiscutable, mais le décapage à l'aide d'une charrue à deux versoirs symétriques présente, en contrepartie du contrôle de la végétation herbacée, l'inconvénient d'installer les plants dans des sillons qui se gorgent d'eau en hiver. II apparaît préférable de pratiquer d'abord le sous-solage, ensuite un billonnage au moyen de disques sur les lignes mêmes de sous-solage ; cette technique a récemment été employée avec succès sur le même terrain.

\section{Choix des essences}

On a longtemps considéré que le pin noir d'Autriche était la seule essence de reboisement susceptible de s'adapter aux terrains comportant une forte proportion de calcaire actif. II s'avère que le pin de Calabre et le pin de Pallas lui sont supérieurs, et le laricio de Corse lui-même, malgré une reprise difficile entraînant un nombre de plants à l'hectare plus faible, assure une production en volume supérieure à celle du pin noir. A ce jour, aucune trace de chlorose n'est apparue sur ces différents pins.

\section{Age des plants}

Les expériences sont antérieures à la normalisation des plants de reboisement pour le fonds forestier national. Le seul critère pris en considération a donc été l'âge des plants. Sur ce point, l'expérience a été probante ; elle conduit à écarter les 1-0 qui n'ont pas permis d'obtenir 
des peuplements valables. Les 1-1 eux-mêmes ne donnent pas des résultats pleinement satisfaisants et, en définitive, dans les conditions climatiques du Centre et du Centre-Est de la France, ce sont les plants repiqués de 3 ans (1-2 ou, mieux peut-être, 2-1) qui doivent être préconisés ; encore est-il nécessaire de leur apporter, au moment de la plantation, tout le soin qu'ils exigent.

\author{
CENTRE TECHNIQUE DU GÉNIE \\ RURAL DES EAUX ET DES FORÉTS \\ Groupement de Nogent-sur-Vernisson \\ Division "Loisirs et chasse" \\ Domaine des Barres \\ 45290 NOGENT-SUR-VERNISSON
}

\title{
RECTIFICATIF A L'ARTICLE : TRAITEMENT CONTRE LE DESSĖCHEMENT DE PLANTS DE PIN LARICIO ET DE PIN SYLVESTRE DESTINÉS AU REBOISEMENT (N 4-1973, PAGE 314 ).
}

Nous indiquions dans cet article que le SUNOCO-FOLICOTE, fabriqué par la SUN OLL Co, n'était pas commercialisé en France. Ceci était exact lorsque l'article nous a étè transmis en Fèvrier 1973. L.a So ciété SEPPIC (Société d'Exploitation de produits pour les Industries Chimiques - 70 Champs Elysées 75008 PARISI nous signale que ce produit est maintenant diffusé en France par ses soins; le prix est de l'ordre de $25 / 30 \mathrm{~F}$ le litre.

\section{ARTICLES PARUS DANS INFORMATIONS TECHNIQUES DU C.T.G.R.E.F}

Cahier $n^{\circ} 11$, Septembre 1973

"La nouvelle règlementation du commerce des graines et des plants forestier ".

Justification de la règlementation découlant de la loi du 22 mai 1971, mise en relief des dispositions prin ciaples qui en résultent, conséquences pour l'Administration, les producteurs et les utilisateurs.

C.T.G.R.E.F. - Groupement de Nogent-s/Vernisson - Division « Graines et plants forestiers ».

"La surveillance des ponts - Réflexions à propos d'une instruction de la Forestry Commission britannique ".

Commentaire d'une instruction de la Forestry Commission britannique dëfinissant les modalités et la périodicité des visites d'inspection des ouvrages d'art et la tenue de fiches signalétiques et de comptes rendus d'inspection.

C.T.G.R.E.F. - Groupement de Nogent-s/Vernisson - Division "Equipement et exploitation des forêts ". 